\title{
One-Stage Reconstruction of an Upper Part Defect of the Auricle
}

\author{
Kotaro Yoshimura, M.D., Takashi Nakatsuka, M.D., Shigeru Ichioka, M.D., Nobuyuki Kaji, M.D., and
} Kiyonori Harii, M.D.

Tokyo, Japan

\begin{abstract}
A one-stage procedure for the reconstruction of a defect of the upper auricle is described. The anterior surface of a carved costal cartilage graft was covered with an anterosuperiorly based skin flap, and the posterior surface was covered by the superficial mastoid fascial flap and a skin graft. This method can be performed easily, without leaving any scar in the hair-bearing area or visible postauricular region, and can be applied to cases in which the condition of the margin scar of an auricular defect is poor.
\end{abstract}

Key words: Auricle—Reconstruction-Mastoid fascia-Onestage-Defect

For the reconstruction of acquired defects of the auricle, a number of two-stage surgical techniques have been described $[2,4,5,11]$. Also, one-stage techniques have been reported for specific cases. A composite graft can be performed for small defects [8], and advancement of auricular skin and cartilage can be applied to helical defects [1]. Davis developed a technically demanding procedure for defects of the upper third that can be performed when a large concha exists [2]. Chen et al. [3] developed a one-stage method with a lengthened postauricular-mastoid flap, which can be used for severe defects, but it is restricted to cases in which a good blood supply across the scar of the defect is preserved. A small helical defect can be repaired by an advancement retroauricular flap [6].

We report a one-stage reconstruction of a defect of the upper third of the auricle using the superficial mastoid

Correspondence to Dr. K. Yoshimura, Department of Plastic and Reconstructive Surgery, University of Tokyo, 7-3-1, Hongo, Bunkyo-Ku, Tokyo 113, Japan. fascial flap and a skin graft. This one-stage technique can be performed even in cases in which the condition of the margin scar is poor, without leaving any scar in the visible mastoid region.

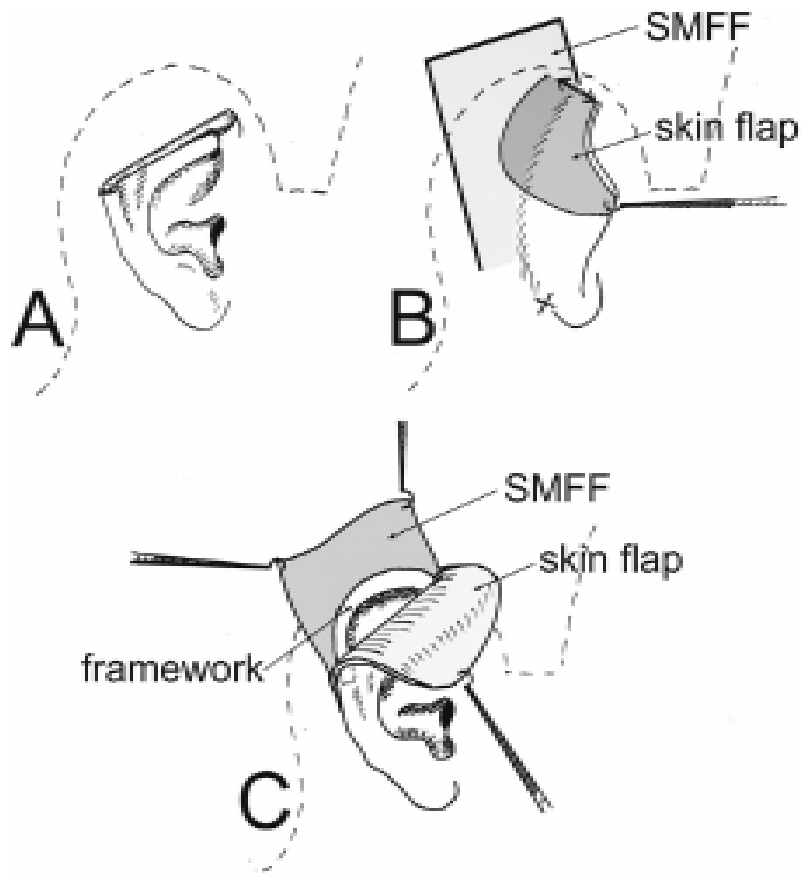

Fig. 1. (A) Preoperative anterior view of an auricular defect (schema). (B) Design of the anterosuperiorly based skin flap and the superficial mastoid fascial flap (SMFF). Note that the pedicle shown by a double arrow $(10 \mathrm{~mm}$ wide $)$ of the skin flap includes the anterior end of the auricular sulcus and the axis of SMFF is directed to the inferior end of the auricle. (C) The framework is placed and covered with the SMFF and the skin flap. 


\section{Surgical Technique}

According to the size and shape of the auricular defect, the anterosuperiorly based skin flap is designed on the posterior aspect of the auricle (Figs. 1A and B). This flap can be extended to the mastoid region, if necessary. A $10-\mathrm{mm}$ width of the anterior end of the auricular sulcus should be included as a pedicle. The anteroinferiorly based superficial mastoid fascial flap is designed on the mastoid and temporal region, with its axis directed to the inferior end of the auricle including the postauricular artery, also according to the defect size (Fig. 1B). The anterosuperiorly based skin flap and the anteroinferiorly based superficial mastoid fascial flap both are raised. A carved costal cartilage framework is then placed on the defect in the auricular cartilage (Fig. 1C) and the posterior and lateral surface of the framework is wrapped with the superficial mastoid fascial flap. The anterior surface of the framework is covered with the anterosuperiorly based skin flap, and the exposed fascial flap of the posterior side is covered with a full-thickness skin graft from the inguinal region. The donor site of the anterosuperiorly based skin flap is repaired by advancing the temporal skin inferiorly with a minimal dog-ear.

\section{Case Report}

The upper posterior part of the right auricle of a 25-yearold man was completely avulsed when he was riding in an automobile with his head out of the window 10 years before his first visit to our hospital (Fig. 2). Since the skin of the scar was very thin, the blood supply across the scar was not expected to be reliable. An anterosuperiorly based skin flap was designed on the posterior aspect of the auricle, and the anteroinferiorly based superficial mastoid fascial flap on the mastoid area (Fig. 3). The anterosuperiorly based skin flap and the anteroinferiorly based superficial mastoid fascia were raised (Fig. 4). After the framework carved out of the costal cartilage was placed, its posterior and lateral surface was completely wrapped with the superficial mastoid fascial flap (Fig. 5). The anterior surface of the framework was then covered with the anterosuperiorly based skin flap, and a full-thickness skin graft from the inguinal region was grafted on the exposed fascial flap followed by a tie-over compression on the grafted skin and a bolster compression to the helix (Fig. 6). Six months after surgery, the good contour was maintained (Fig. 7). Secondary correction of the dog-ear deformity of the skin flap pedicle was not performed.

\section{Discussion}

The one-stage reconstruction of microtia with the superficial and deep mastoid fascias was introduced by Song and Song [13] and modified and refined by Park et al. $[9,10]$. Park et al. [9] have also performed an anatomical cadaver study of the subcutaneous fascial layers in the mastoid region. The superficial mastoid fascia used in this paper corresponds to the superficial temporal fascia cephally [9]. The superficial mastoid fascia, which is supplied primarily by the posterior auricular artery, can be elevated in three ways: (i) the anteroinferiorly based flap supplied by the posterior auricular artery [13], (ii) the posteroinferiorly based flap supplied by the anastomoses between the posterior auricular artery and the occipital artery [12], and (iii) the superiorly based flap supplied by the anastomoses between the posterior auricular artery and the posterior branch of the superficial temporal artery or the superior auricular artery $[7,14]$. For various types of the auricle reconstruction, the superficial mastoid fascia can be utilized instead of the temporoparietal fascial flap, which cannot be performed without leaving a visible scar in the hair-bearing area.

An anterosuperiorly based postauricular skin flap used here could be elevated with a narrow skin pedicle, e.g., $10 \mathrm{~mm}$ wide. The superior auricular artery [14] is presumed to support or even nourish this narrow and long flap as a nearly axial pattern flap. The abundant blood supply of this flap enables the one-stage procedure without the secondary correction of the dog-ear of the skin pedicle. In accordance with the size of the auricular defect, the anterosuperiorly based flap can be extended from the posterior aspect of the auricle to the mastoid area.

Chen et al. [3] introduced a one-stage method for auricular defects using the postauricular-mastoid extended flap distally with a piece of the occipital subcutaneous tissue. This flap, however, relies on the vascular connection between the preauricular and the postauricular blood vessels across the scar of the defect margin, so that this method cannot be used in cases in which the condition of the scar is poor. Another drawback is the large visible scar in the mastoid region. In contrast, the method proposed by us can be applied to any case regardless of the condition of the marginal scar, and the bolster compression, if preferred, can be performed for better contouring of the helix. Although our method can be used only for upper-third or half defects of the auricle, it can be modified and applied to any auricular defects if the blood supply across the margin scar of the defect is reliable. In such cases, the skin flap for the anterior surface can be designed according to the defect size with the margin of the defect as a pedicle, and the posterior surface can be repaired with the superficial mastoid fascial flap and a skin graft, without leaving any scar in the visible mastoid region.

\section{References}

1. Antia NH, Buch VI: Chondrocutaneous advancement of flap for the marginal defect of the ear. Plast Reconstr Surg 39:472, 1967

2. Brent B: Reconstruction of the auricle. In: McCarthy JG, ed. Plastic surgery. Saunders: Philadelphia, 1990, p 2094

3. Chen Z, Chen C, Zhang J: Improved technique for a onestage repair of significant defects of the ear. Plast Reconstr Surg 86:987, 1990 

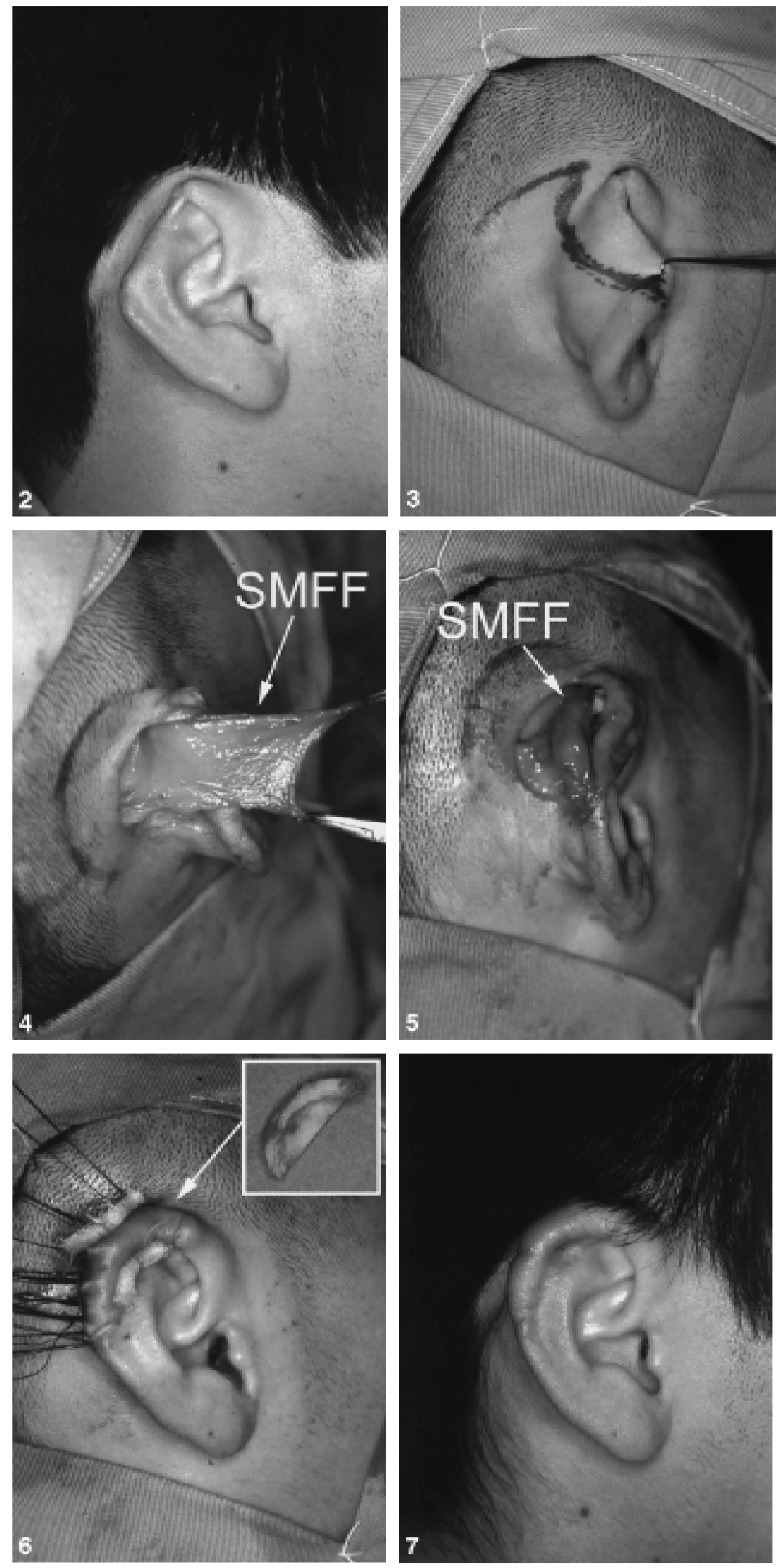

Fig. 2. A preoperative view of the auricle of a 25 -year-old man.

Fig. 3. Design of the anterosuperiorly based skin flap. An additional small incision was made for easy elevation of the superficial mastoid fascial flap.

Fig. 4. The superficial mastoid fascial flap (SMFF) is elevated.

Fig. 5. A carved cartilage framework is placed, and its posterior and lateral surface is wrapped with the superficial mastoid fascial flap (SMFF).

Fig. 6. A bolster compression is applied to the helix. The inset shows the carved cartilage framework.

Fig. 7. A postoperative view 6 months after surgery. 
4. Converse JM: Reconstruction of the auricle-part 1. Plast Reconstr Surg 22:150, 1958

5. Converse JM: Reconstruction of the auricle-part 2. Plast Reconstr Surg 22:230, 1958

6. Kaminsky A: Repair of the partial loss of the helix. Aesth Plast Surg 21:427, 1997

7. Kobayashi S, Yoza S, Kakibuchi M, Sekiguchi J, Ohmori $\mathrm{K}$ : Retroauricular hairline flap transfer to the face. Plast Reconstr Surg 96:42, 1995

8. Nagel F: Reconstruction of a partial auricular loss. Plast Reconstr Surg 49:340, 1972

9. Park C, Lee TJ, Shin KS, Kim YW: A single-stage twoflap method of total ear reconstruction. Plast Reconstr Surg 88:404, 1991
10. Park C: Modification of two-flap method and framework construction for reconstruction of atypical congenital auricular deformities. Plast Reconstr Surg 99:1846, 1997

11. Quaba A: Reconstruction of a posttraumatic ear defect using tissue expansion: thirty years after Neumann. Plast Reconstr Surg 82:521, 1988

12. Sharma RK, Kobayashi K, Jackson IT, Carls FR: Vascular anatomy of the galeal occipitalis flap: a cadaver study. Plast Reconstr Surg 97:25, 1996

13. Song Y, Song Y: An improved one-stage total ear reconstruction procedure. Plast Reconstr Surg 71:615, 1983

14. Song R, Song Y, Qi K, Jiang H, Pan F: The superior auricular artery and retroauricular arterial island flaps. Plast Reconstr Surg 98:657, 1996 